\title{
Stochastic Lattice gas Cellular Automata Model for Epidemics
}

\author{
Ariel Félix Gualtieri and Juan Pedro Hecht \\ Department of Biophysics, Faculty of Dentistry, University of Buenos Aires, Buenos Aires C1122AAH, Argentina
}

\begin{abstract}
The aim of this study was to develop and explore a stochastic lattice gas cellular automata (LGCA) model for epidemics. A computer program was development in order to implement the model. An irregular grid of cells was used. A susceptible-infected-recovered (SIR) scheme was represented. Stochasticity was generated by Monte Carlo method. Dynamics of model was explored by numerical simulations. Model achieves to represent the typical SIR prevalence curve. Performed simulations also show how infection, mobility and distribution of infected individuals may influence the dynamics of propagation. This simple theoretical model might be a basis for developing more realistic designs.
\end{abstract}

Key words: Disease spread, people movement, epidemic model, stochastic lattice gas cellular automata.

\section{Introduction}

In the large majority of studies involving population dynamics, such as the spread of epidemics, direct experimentation is often not feasible. That is why formal models can offer a great help, providing a framework for exploring these scenarios. Mathematical models of epidemics are formal designs that capture the dynamic behaviour of spread of infectious diseases [1, 2].

The first step to define an epidemic model is to classify individuals of the population into different categories corresponding to possible states for the disease under study. According to this grouping, parameters are defined to represent the transition of individuals between these categories over a period of time. The aim then is to study the evolution of the system over time [3]. The choice of which states to include in a model depends on the characteristics of the particular disease being investigated and the purpose of the model. Acronyms for epidemic models are often based on the flow patterns between the different states considered [4]. For example, in a

Corresponding author: Ariel Félix Gualtieri, Ph.D., research fields: applied mathematics, epidemiology and biostatistics. susceptible-infected-recovered (SIR) type model, individuals can be in one of three states; they are susceptible (S) and can catch the disease, infected (I) and can spread the disease, or recovered and immune (R). The SIR scheme is representative of diseases in which individuals develop immunity, such as influenza [5-7].

The dynamics of current models of epidemics rarely lead to analytical solutions. To achieve this, it would be necessary simplifications to reduce the formal complexity of the models, which would reduce their representativity [8]. Thus, despite its formal nature, the results provided by current epidemiological mathematical models are often analyzed in a qualitative way from numerical computer simulations. The difficulty of including all relevant factors, the imprecise measurement of biological and behavioural variables, and the extreme sensitivity of many non-linear systems to small changes in parameter values are frequently insurmountable obstacles to accurate quantitative prediction [9]. Thus, qualitative results can provide a coherent framework of analysis that is more efficient than simple intuition, helping in the planning of health policies.

Both deterministic and stochastic models are used 
to describe the transmission dynamics of epidemics. The deterministic models based in systems of equations, often lead to powerful qualitative results with important threshold behaviour. They also lead to simpler mathematical problems than the stochastic ones. Work on deterministic models has therefore dominated strongly over work on stochastic models [10]. However, deterministic models are not appropriate when population size is small and stochastic factors play a major role [9]. There are a number of ways to allow the events in a model to be influenced by chance, but the most common and rigorous method is Monte Carlo simulation, where a set of possible events is defined with a probability attached to each of them. A random number generator is then used to calculate which of the range of possible events it will be [9].

Classical epidemic models have tended to minimize geographic heterogeneity and related spatial aspects of spread of infectious diseases. However, spatial epidemic models have become more important in recent years [11-13]. Spatial models of infectious disease transmission provide the only framework in which knowledge of the location of hosts and their movement patterns can be combined with a description of the infection process and the disease natural history to investigate observed patterns and to evaluate alternative intervention options [14].

The significant development of computer technology during the last decades has allowed the design of mathematical computer models that supply explicit spatial representations of complex systems. One example is the cellular automata (CA) models. They are dynamic simulation models defined by spatially arranged mathematical cells, which are updated in discrete steps according to a set of rules. The nature of these update rules determines whether the model will have a deterministic or a stochastic behaviour [15]. In recent years the development of CA models for the spread of infectious diseases has increased. Both deterministic [16-18] and stochastic [19-21] designs have been developed.

In CA models for epidemics, each cell can be considered as an individual or a small subpopulation [22-24]. Alternatively, each cell may represent an area (patch) occupied by a certain number of individuals who can interact within the cell and move to other cells $[17,19,25]$. This will be the case in the model presented in this work. A CA whose cells contain particles that interact within the cells is called lattice gas cellular automata (LGCA) [19]. As computer technology continues to improve, the development of epidemiological models of CA also increases. Although these models do not provide analytical solutions, they help to understand the spatial dynamics and to identify what factors might be relevant to the spread of epidemics in spatially structured populations [26].

The aim of the present work is to develop and explore a stochastic LGCA model that can be a basis for studying the spread and control of epidemics and their relation to the spatial distribution and mobility of the population.

\section{Materials and Methods}

The model was implemented in an original computer program developed by the authors of the present work in Delphi ${ }^{\circledR}$ platform. The software includes the Delphi-Component Tmesh for representing irregular grids in cellular automata simulations [27]. The model presents a two-dimensional grid of 900 cells with different shapes incorporated in this component (Fig. 1). If two cells are adjacent, they are considered neighbours. Thus, each cell can be surrounded by a different number of neighbours. In each cell there is a given number of individuals who can move between neighbouring cells.

A SIR scheme has been represented. That is, as mentioned in the introduction, individuals were classified into three successive states: susceptible (S), infected (I) 


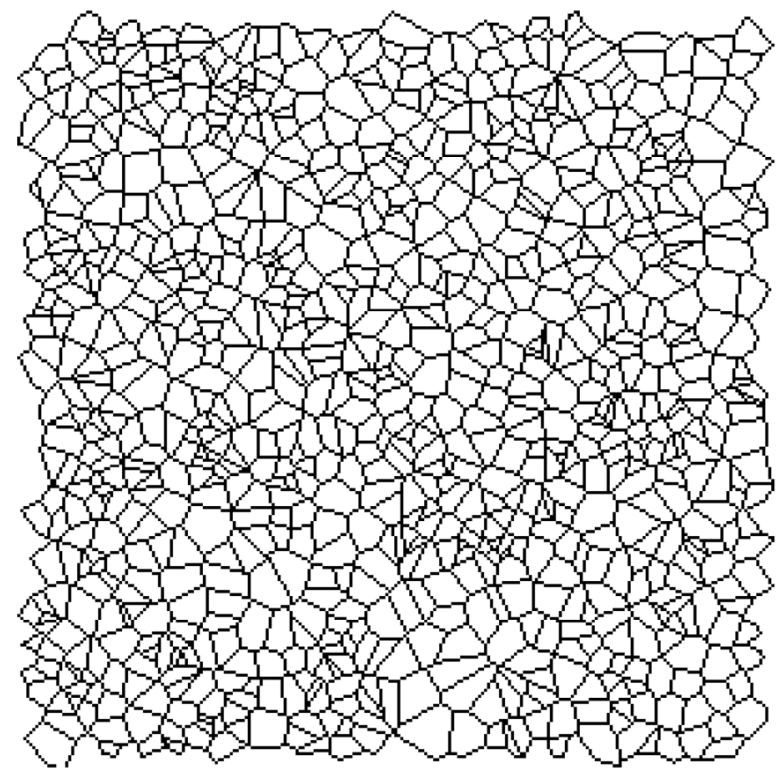

Fig. 1 Two-dimensional grid of 900 cells included in the Delphi-Component Tmesh [27] and used in the model of the present work.

or recovered (R). For each cell $i$, the model includes the following events influenced by chance: infection, recovery and displacement to a neighbouring cell selected at random. A state transition parameter is assigned to each of these events. The authors named $\beta$, $\gamma$ and $\delta$ to parameters for infection, recovery and displacement, respectively. These parameters have the same value in all cells. The value of each parameter is proportional to the probability of occurrence of the corresponding process. In addition, it is considered that the net rate at which infection is acquired in each cell $i$ also depends on the number of encounters between susceptible and infected in that cell. Thus, for each cell $i$ the net rate of infection is proportional to $\beta S_{i} I_{i}$, where $S_{i}$ and $I_{i}$ are the number of susceptible and infected individuals placed in the cell $i$, respectively.

The system is updated at discrete intervals of time (epochs), incorporating random effects by using Monte Carlo method according to the manner described below. The parameters that affect individuals of each state define a range proportional to its magnitude in the interval $(0,1)$. For each epoch a random number generator built into the program produces values within this range, which allows selecting the event that individuals of each state will experience. Thus, for each epoch, individuals can experience one of the following events: change of state, displacement to a neighbouring cell selected at random or remain unchanged. Finally, the number of individuals in each state per cell is updated and the simulation time is incremented by one epoch. The program allows to set the initial number of individuals in different states by cell. When a simulation is completed, the program generates an analytical record of the number of individuals in the states $\mathrm{S}, \mathrm{I}$ and $\mathrm{R}$ for each cell and for the entire system as a function of simulation time in epochs. The application also provides visual representations of the spread of the outbreak on the lattice of cells through bitmaps.

The dynamics of the model was explored by computer numerical simulations. Arbitrary values were assigned to initial conditions and to state transition parameters.

\section{Results}

The authors considered a benchmark case with the following initial distribution of people on the gird: one infected individual in a specific cell, twenty-five susceptible individuals in each cell and zero recovered individual in the system. The parameters were set to $\beta$ $=0.0075, \gamma=0.0150$ and $\delta=0.1500$. Fig. 2a shows the obtained prevalence curve based on 10 repetitions of a simulation of 400 epochs. These repetitions follow a common pattern but they are not identical because of the influence of chance on infection, recuperation and displacement. Thus, the authors represented the mean and the standard deviation of the proportion of infected individuals in each epoch. The obtained prevalence curve follows the expected behaviour for a SIR model. That is, the proportion of infected individuals increases exponentially to a maximum value and then begins to decrease. A typical series of bitmaps generated in one of these repetitions is illustrated in Fig. 2b. Bitmaps represent the spatial pattern of the infected individuals for different times, by using a gray scale described in 


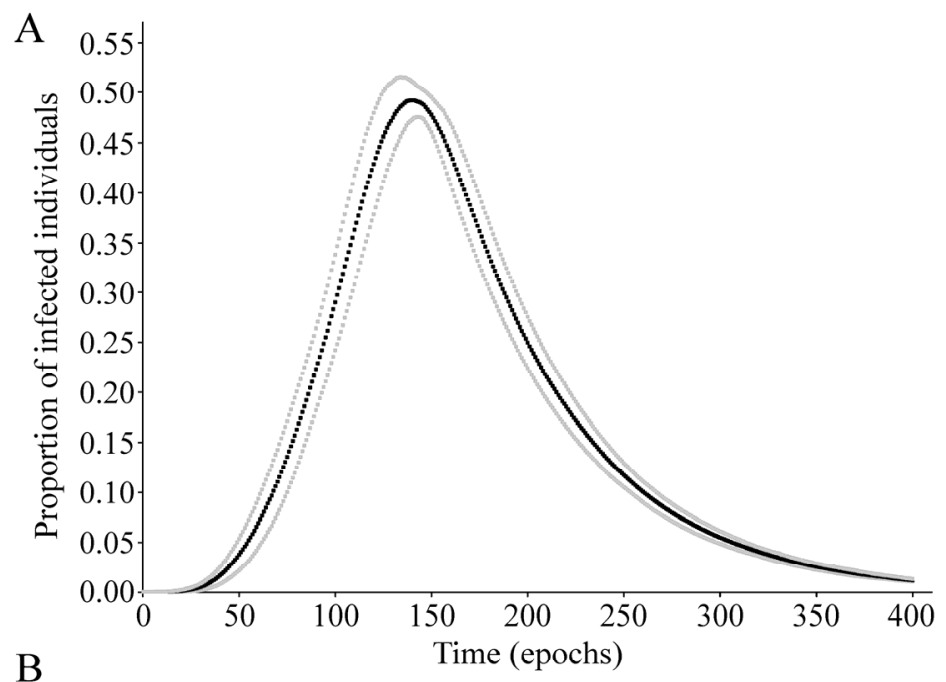

B

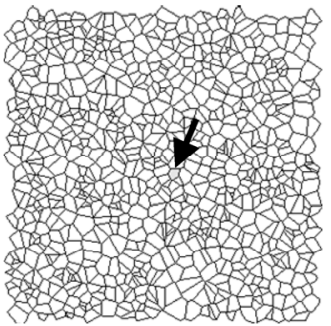

epoch 0

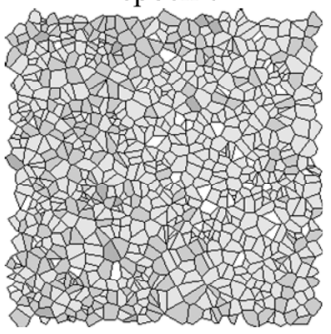

epoch 225

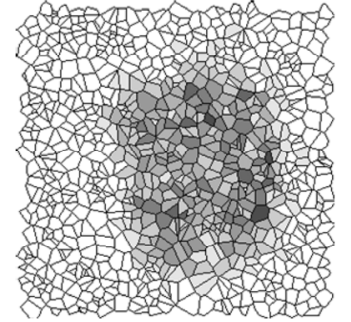

epoch 75

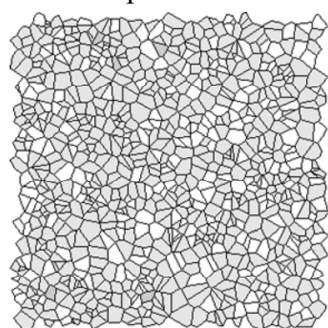

epoch 300

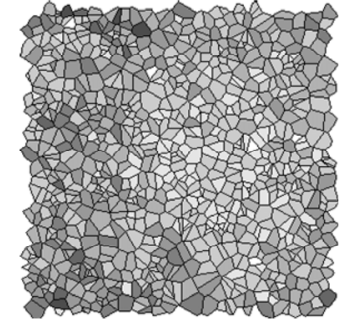

epoch 150

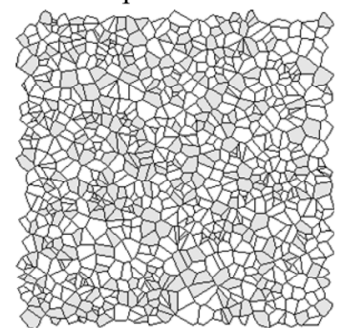

epoch 375

Fig. 2 Evolution of the epidemic in the benchmark case. (A) Proportion of infected individuals as a function of time. Mean (black markers) and mean \pm standard deviation (gray markers) of 10 repeat simulations are displayed. (B) Spatial pattern of the infected individuals for different times in a typical simulation. The colour of each cell is related to the number of infected individuals placed there: white correspond to zero infected individuals and a darker shade of gray indicates a larger number of them. The arrow indicates the cell in which infected individuals are placed at epoch 0.

the figure legend. It can be seen how the evolution of epidemics on the lattice is in agreement with the obtained prevalence curve.

The authors explored the sensitivity of the system to parameters $\beta$ and $\delta$ by varying them one by one, and keeping other conditions as in the benchmark case. These parameters might be affected by health control strategies. For example, some measures implemented during an influenza epidemic can cause a direct reduction in the rate of infection of the disease, such as antiviral prophylaxis or promotion of hand and respiratory hygiene [28, 29]. Fig. 3 shows the evolution of prevalence for different values of $\beta$. As expected, the peak prevalence reduces with decreasing $\beta$. The restriction of population mobility is another strategy that can be adopted to mitigate an influenza epidemic [30]. Accordingly, in Fig. 4 the authors show how a reduction of the parameter $\delta$, also produces a decrease in the maximum of prevalence. 


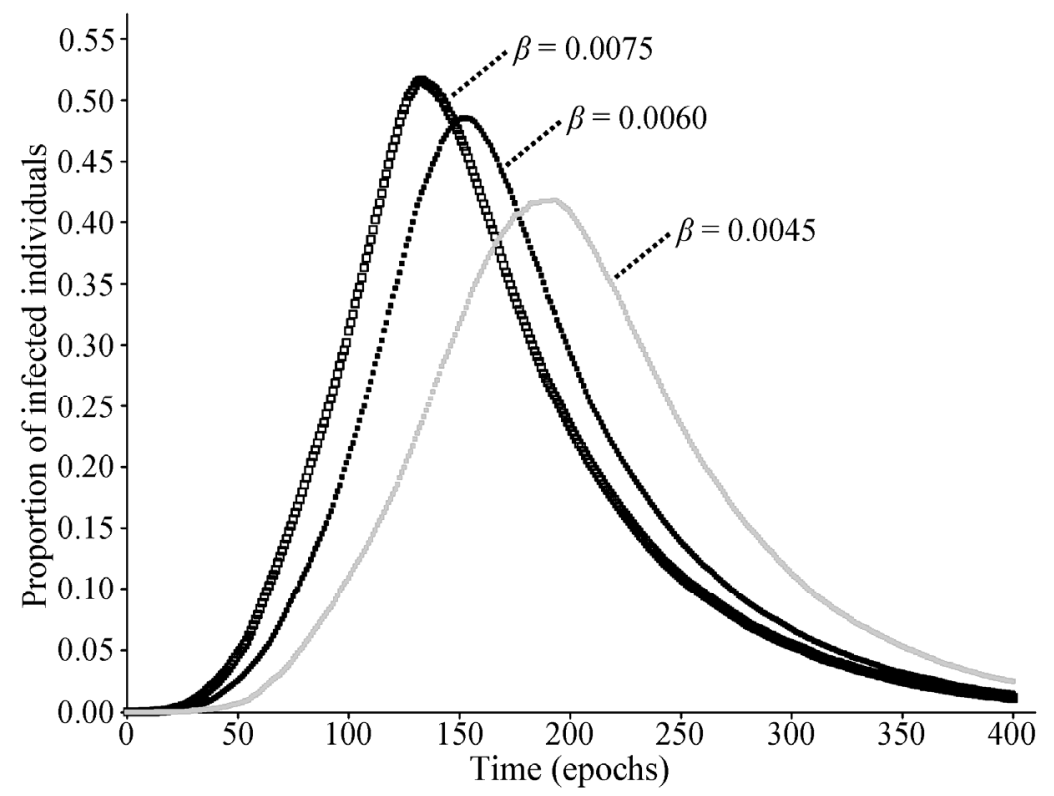

Fig. 3 Influence of $\beta$ on the evolution of prevalence. Three values were tested: $\beta=0.0075$ (open black markers); $\beta=0.0060$ (solid black markers); $\beta=0.0045$ (solid gray markers). Other conditions remain the same as the benchmark case. A typical simulation is shown for each value.

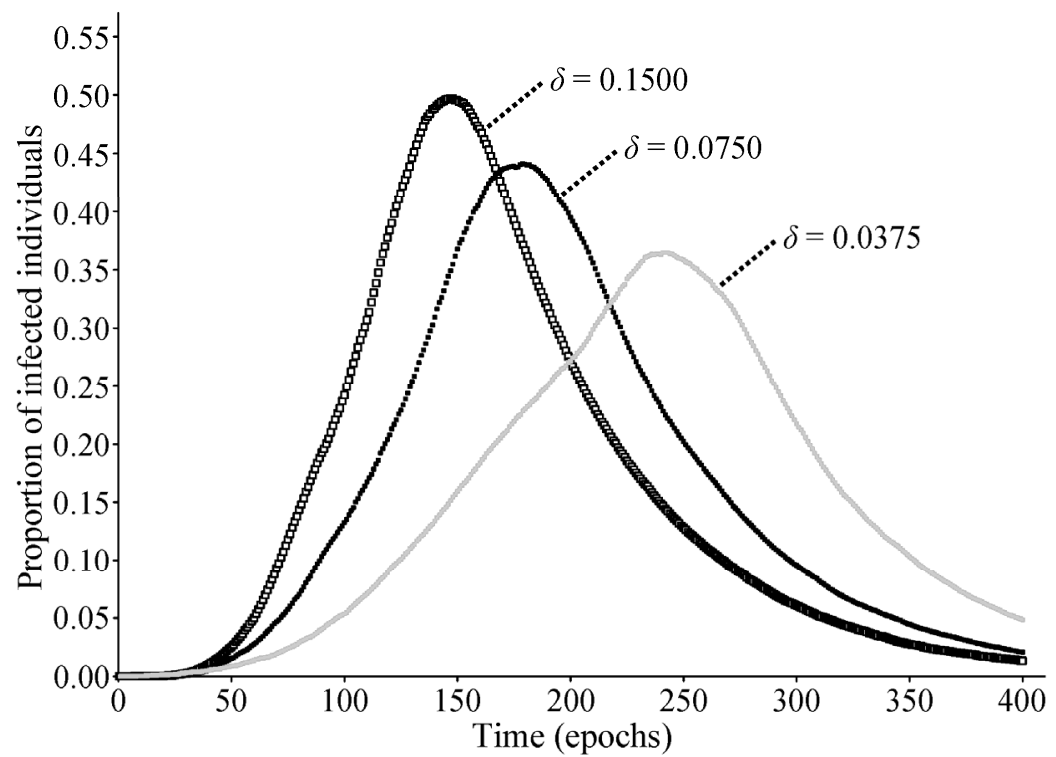

Fig. 4 Influence of $\delta$ on the evolution of prevalence. Three values were tested: $\delta=0.1500$ (open black markers); $\delta=0.0750$ (solid black markers); $\delta=\mathbf{0 . 0 3 7 5}$ (solid gray markers). Other conditions remain the same as the benchmark case. A typical simulation is shown for each value.

Finally, the authors show that the initial distribution of infected individuals can influence the behaviour of the system. Two initial scenarios were compared: a central cell occupied by six infected individuals and two cells with three infected individuals in each one (Fig. 5a); all other conditions remained as in the benchmark case. The prevalence curve is more steep during the ascending phase and a higher peak prevalence is reached when infected individuals are distributed in two cells than when they are located in one cell at the beginning of the simulation (Fig. 5b).

\section{Discussion}

The authors developed a model which could be the 


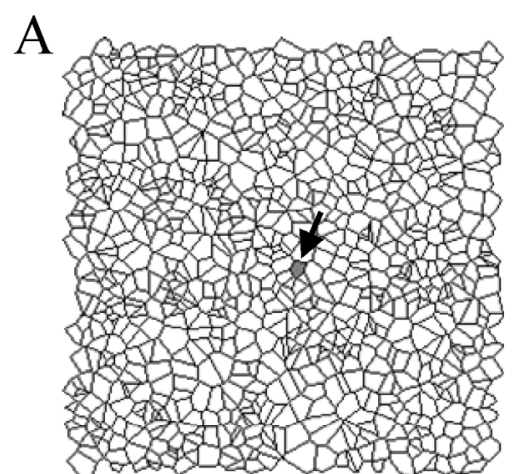

Scenario 1

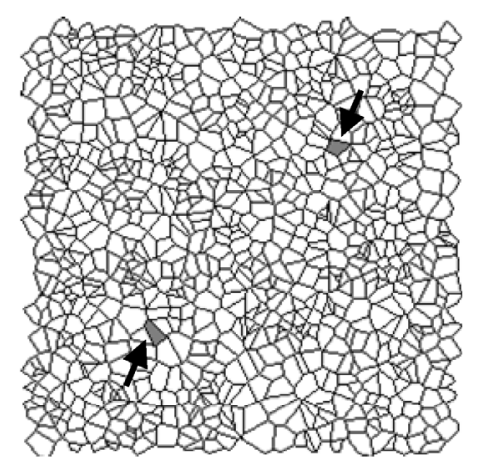

Scenario 2

$\mathrm{B}$

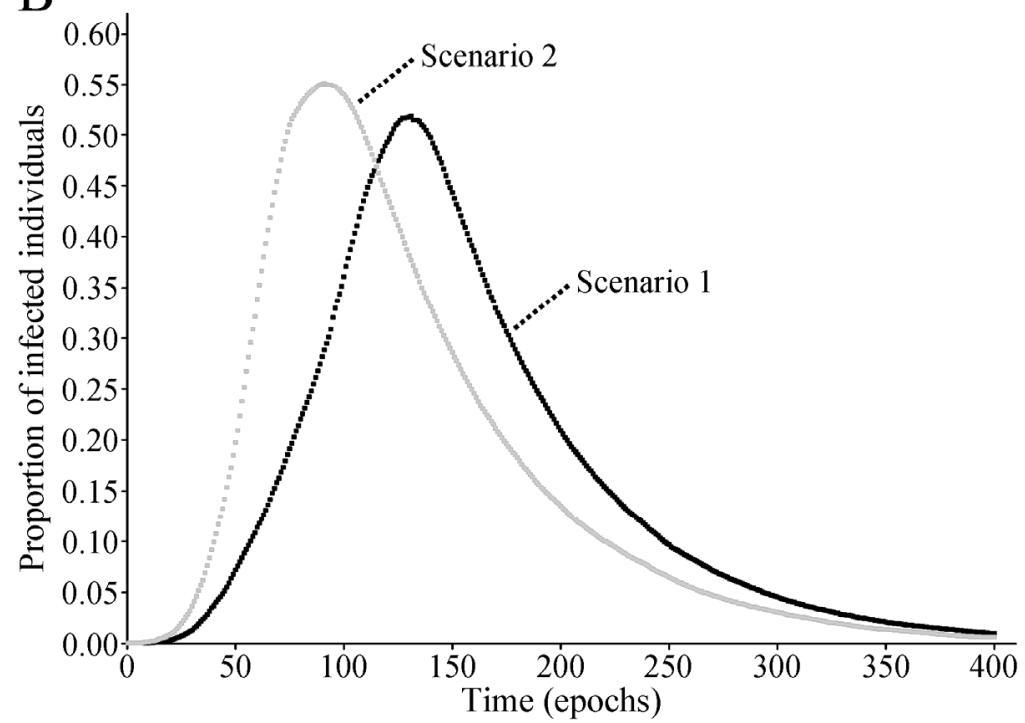

Fig. 5 Influence of the initial distribution of infected individuals on the evolution of prevalence. Two initial scenarios were compared. Scenario 1: a central cell occupied by six infected individuals. Scenario 2: two cells with three infected individuals in each one. (A) Visual representation of each scenario at epoch 0 . The arrows indicate the cells in which infected individuals are placed at epoch 0 . (B) Typical prevalence curves obtained to scenario 1 (black markers) and scenario 2 (gray markers).

theoretical basis for the study of different scenarios where it is important to assess the mobility and distribution of individuals among several areas. For example, it could be an initial design for the investigation of the spread of outbreaks of some diseases (e.g., influenza) in megacities, where the restriction on population mobility could be a public health measure implemented by governments [30]. Each cell in the system (patch) could represent a given area of the city. On the other hand, the model allows to set the initial distribution of infected individuals, which could be a point of interest for governments in order to evaluate the impact of bioterrorist attacks in megacities. In recent years, bioterrorism has increased the interest in the development of mathematical models of spread of infectious diseases [31-33].

A feature of the developed model is that it achieves represent a stochastic dynamics. This is evident because for the same set of parameter values and initial conditions the authors get similar but not identical outputs. In population phenomena, such as the spread of infectious diseases, stochastic effects can be relevant. Therefore, the inclusion of stochasticity in an epidemic model can give it more realism. Cellular automata models where individuals move randomly in their neighborhood have been developed [19, 34, 35]. However, randomness for other events is often ignored. The LGCA model development in the present 
work incorporates stochastic effects for mobility and, in addition, for infection and recovery, achieving the usual SIR prevalence curve. Infection and recovery are biological processes and it is reasonable to assume that they may be influenced by chance.

Other models in the literature present grids made up of identical cells (usually square shaped) arranged uniformly. They have classical systems of neighborhood such as the neighborhood of Moore, which consists of the cell itself and its eight nearest neighbor cells [18, 23, 24]. However, Flache and Hegselmann have suggested that cellular automata dynamics could have general properties that are robust to variation in the grid structure [27]. Irregular neighborhood system used in the present model can incorporate more spatial heterogeneity, which could be closer to real conditions.

Although the model developed in the present work succeeds in capturing qualitatively a typical SIR dynamic, it is a relatively simple design and may be complexified in different ways in order to incorporate more realism.

\section{Conclusions}

The authors have developed a stochastic susceptible-infected-recovered (SIR) epidemic model of lattice gas cellular automata (LGCA). The general behaviour of the theoretical prevalence curves obtained in the model coincides with the propagation dynamics of diseases that can be represented by the SIR scheme, like influenza. In addition, the model responds as expected to variations in infection rate and mobility. Since, as might be supposed, the peak prevalence increases with the rate of infection and mobility. The complexification of this model to adapt it to any particular real scenario might be the subject of a future work.

\section{Acknowledgments}

The authors gratefully acknowledge the University of Buenos Aires for providing financial support (grant numbers UBACyT O405, UBACyT 20020090200258, UBACyT 20020130100417BA).

\section{References}

[1] Anderson, R. M., and May, R. M. 1988. Infectious Diseases of Humans: Dynamics and Control. New York: Oxford University Press.

[2] Siettos, C. I., and Russo, L. 2013. "Mathematical Modeling of Infectious Disease Dynamics." Virulence 4 (4): 295-306.

[3] Ferguson, N. M. 2005. "Mathematical Prediction in Infection." Medicine 33 (3): 1-2.

[4] Hethcote, H. W. 2000. "The Mathematics of Infectious Diseases." Society for Industrial and Applied Mathematics Review 42 (4): 599-653.

[5] Laguzet, L., and Turinici, G. 2015. "Individual Vaccination as Nash Equilibrium in a SIR Model with Application to the 2009-2010 Influenza A (H1N1) Epidemic in France." Bulletin of Mathematical Biology 77 (10): 1955-84.

[6] Vaidya, N. K., Morgan, M., Jones, T., Miller, L., Lapin, S., and Schwartz, E. J. 2015. "Modelling the Epidemic Spread of an H1N1 Influenza Outbreak in a Rural University Town." Epidemiology and Infection 143 (8): 1610-20.

[7] Huang, X., Clements, A. C., Williams, G., Mengersen, K., Tong, S., and Hu, W. 2016. "Bayesian Estimation of the Dynamics of Pandemic (H1N1) 2009 Influenza Transmission in Queensland: a space-time SIR-based model." Environmental Research 146 (4): 308-14.

[8] Cassels, S., Clark, S. J., and Morris, M. 2008. "Mathematical Models for HIV Transmission Dynamics: Tools for Social and Behavioral Science Research." Journal of Acquired Immune Deficiency Syndromes 47 (1): S34-9.

[9] Garnett, G. P. 2002. "An Introduction to Mathematical Models in Sexually Transmitted Disease Epidemiology." Sexually Transmitted Infections 78 (1): 7-12.

[10] Nåsell, I. 2002. "Stochastic Models of Some Endemic Infections." Mathematical Biosciences 179 (1): 1-19.

[11] Gualtieri, A. F., and Hecht, J. P. 2013. "Simulation of Spread of Infectious Diseases and Population Mobility in a Deterministic Epidemic Patch Model." Journal of Life Sciences 7 (3): 252-8.

[12] Li, L. 2015. "Patch Invasion in a Spatial Epidemic Model." Applied Mathematics and Computation 258 (5): 342-9.

[13] Cai, Y., and Wang, W. 2016. "Fish-hook Bifurcation Branch in a Spatial Heterogeneous Epidemic Model with Cross-diffusion." Nonlinear Analysis: Real World Applications 30 (8): 99-125. 
[14] Riley, S. 2007. "Large-scale Spatial-transmission Models of Infectious Disease." Science 316 (5829): 1298-301.

[15] Venkatachalam, S., and Mikler, A. R. 2006. "An Infectious Disease Outbreak Simulator based on the Cellular Automata Paradigm." In Innovative Internet Community Systems, edited by Böhme, T., Larios Rosillo, V. M., Unger, H., and Unger, H. Berlin-Heidelberg: Springer.

[16] Sun, G. Q., Liu, Q. X., Jin, Z., Chakraborty, A., and Li, B. L. 2010. "Influence of Infection Rate and Migration on Extinction of Disease in Spatial Epidemics." Journal of Theoretical Biology 264 (1): 95-103.

[17] Crawford, B. A., Kribs-Zaleta, C. M., and Ambartsoumian, G. 2013. "Invasion Speed in Cellular Automaton Models for T. cruzi vector Migration." Bulletin of Mathematical Biology 75 (7): 1051-81.

[18] El Yacoubi, S., and Gourbière, S. 2016. "The Spatial Reproduction Number in a Cellular Automaton Model for Vector-borne Diseases Applied to the Transmission of Chagas Disease." Simulation 92 (2): 141-52.

[19] Schneckenreither, G., Popper, N., Zauner, G., and Breitenecker, F. 2008. "Modelling SIR-type Epidemics by ODEs, PDEs, Difference Equations and Cellular Automata-A Comparative Study." Simulation Modelling Practice and Theory 16 (8): 1014-23.

[20] Rehkopf, D., Furumoto-Dawson, A., Kiszewski, A., and Awerbuch-Friedlander, T. 2015. "Spatial Spread of Tuberculosis through Neighborhoods Segregated by Socioeconomic Position: a Stochastic Automata Model." Discrete Dynamics in Nature and Society 583819: 1-8.

[21] da Silva, R., and Fernandes, H. A. 2015. "A Study of the Influence of the Mobility on the Phase Transitions of the Synchronous SIR Model." Journal of Statistical Mechanics: Theory and Experiment 6: P06011.

[22] van Ballegooijen, W. M., and Boerlijst, M. C. 2004. "Emergent Trade-offs and Selection for Outbreak Frequency in Spatial Epidemics." Proceedings of the National Academy of Sciences 101 (52): 18246-50.

[23] Karim, M. F. A., Ismail, A. I. M., and Ching, H. B. 2009. "Cellular Automata Modelling of Hantarvirus Infection." Chaos, Solitons and Fractals 41 (5): 2847-53.

[24] Athithan, S., Shukla, V. P., and Biradar, S. R. 2015. "Voting Rule based Cellular Automata Epidemic Spread
Model for Leptospirosis." Indian Journal of Science and Technology 8 (4): 337-41.

[25] White, S. H., del Rey, A. M., and Sánchez, G. R. 2007. "Modeling Epidemics using Cellular Automata." Applied Mathematics and Computation 186 (1): 193-202.

[26] Bauch, C. T. 2005. "The Spread of Infectious Diseases in Spatially Structured Populations: an Invasory Pair Approximation." Mathematical Biosciences 198 (2): 217-37.

[27] Flache, A., and Hegselmann, R. 2001. "Do Irregular Grids Make a Difference? Relaxing the Spatial Regularity Assumption in Cellular Models of Social Dynamics." Journal of Artificial Societies and Social Simulation 4 (4). http://jasss.soc.surrey.ac.uk/4/4/6.html.

[28] Ferguson, N. M., Cummings, D. A., Fraser, C., Cajka, J. C., Cooley, P. C., and Burke, D. S. 2006. "Strategies for Mitigating an Influenza Pandemic.” Nature 442 (7101): 448-52.

[29] World Health Organization (WHO) 2013. Pandemic Influenza Risk Management WHO Interim Guidance. Geneva: WHO Press.

[30] World Health Organization (WHO) 2010. Pandemic Influenza Preparedness and Response: a WHO Guidance Document. Geneva: WHO Press.

[31] Elderd, B. D., Dukic, V. M., and Dwyer, G. 2006. "Uncertainty in Predictions of Disease Spread and Public Health Responses to Bioterrorism and Emerging Diseases." Proceedings of the National Academy of Sciences 103 (42): 15693-7.

[32] Gonçalves, B., Balcan, D., and Vespignani, A. 2013. "Human Mobility and the Worldwide Impact of Intentional Localized Highly Pathogenic virus Release." Scientific Reports 3 (810): 1-7.

[33] Graeden, E., Fielding, R., Steinhouse, K. E., and Rubin, I. N. 2015. "Modeling the Effect of Herd Immunity and Contagiousness in Mitigating a Smallpox Outbreak." Medical Decision Making 35 (5): 648-59.

[34] Fu, S. C., and Milne, G. 2004. "A Flexible Automata Model for Disease Simulation." Lecture Notes in Computer Science 3305: 642-9.

[35] Liu, Q. X., Jin, Z., and Liu, M. X. 2006. "Spatial Organization and Evolution Period of the Epidemic Model using Cellular Automata." Physical Review E 74 (3): 031110/1-031110/6. 